\title{
Nahrungsmittelallergie: Klinik, Diagnostik und Therapie
}

\section{Food Allergy: Clinical Symptoms, Allergy Diagnostic Tests, Treatment Options}

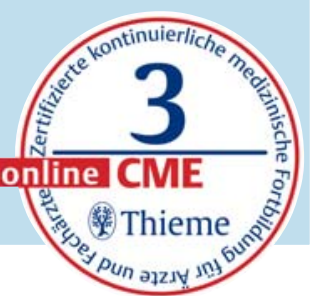

U. Jappe $e^{1,2}$

${ }^{1}$ Klinik für Dermatologie, Allergologie und Venerologie der Universität zu Lübeck

${ }^{2}$ Klinische und Molekulare Allergologie, Forschungszentrum Borstel

VNR

2760512011060000424

Bibliografie

DOI http://dx.doi.org/

10.1055/s-0030-1256478

Akt Dermatol 2011; 37 :

218-230 @ Georg Thieme

Verlag KG Stuttgart · New York

ISSN 0340-2541

Korrespondenzadresse

Prof. Dr. med. Uta Jappe, MSc

Klinik für Dermatolgie,

Allergologie und Venerologie

der Universität Lübeck

Ratzeburger Allee 160

23562 Lübeck

uta.jappe@uk-sh.de

\section{Lernziele}

Kenntnisse über

- Auslöser, Augmentations- und Risikofaktoren einer Nahrungsmittelallergie

- Differenzialdiagnose der immunologisch bedingten Nahrungsmittelallergie

- Diagnostik: Wertigkeit von Hauttestungen und IgE-Detektion

- Sensibilisierungswege und ihr Einfluss auf Symptomatik und Verlauf

- Bedeutung von Kreuzreaktionen und ihre molekularen Grundlagen

- komponentenbasierte IgE-Diagnostik

- Provokationstestungen

- medikamentöse Therapie der Nahrungsmittelallergie

- Stand der spezifischen Immuntherapie von Nahrungsmittelallergien

\section{Einleitung}

$\nabla$

Gemäß Definition ist eine Nahrungsmittelallergie eine immunologisch bedingte Überempfindlichkeitsreaktion mit Ausprägung einer klinischen Symptomatik, welche mit dem Genuss des betreffenden Nahrungsmittels (der betreffenden Nahrungsmittelallergen-Quelle) assoziiert ist. Zumeist handelt es sich hierbei um IgE-Antikörpervermittelte Soforttypreaktionen. Es können aber auch Spätreaktionen auftreten, die sich als hämatogene Kontaktdermatitis oder im Sinne der Verschlechterung eines atopischen Ekzems [1,2] manifestieren können. Die Proteinkontaktdermatitis stellt einen Sonderfall dar [3].

\section{Symptomatik}

$\nabla$

Die Nahrungsmittelallergie ist sehr viel seltener, als es von Patienten selbst angenommen wird. Das Hautorgan ist besonders häufig betroffen. Soforttypreaktionen bestehen dabei vornehmlich aus Symptomen des oralen Allergiesyndroms, der Urtikaria und dem Quinckeödem sowie Pruritus und Flush. Selten kann es zu respiratorischen Symptomen kommen, worunter die Rhinoconjunktivitis allergica und das Asthma bronchiale allergicum am häufigsten sind. Gastrointestinale Symptome bestehen aus Nausea, Vomitus, gastrointestinalen Schmerzen und Diarrhöen [1].

Seltene Folge einer Nahrungsmittelallergie können anaphylaktische Kreislaufreaktionen sein, wobei Todesfälle häufiger durch einen Status asthmaticus als durch anaphylaktische Kreislaufreaktionen bedingt sind [4].

Die Nahrungsmittelallergie kann in jedem Lebensalter auftreten. Im frühen Kindesalter sind im Wesentlichen Hühnerei, Kuhmilch, Soja, Erdnüsse, Baumnüsse, Fisch und Weizen die Ursache. Sie sind die klassischen Nahrungsmittelallergene (Klasse 1). Die pollenassoziierten Nahrungsmittelallergien (Klasse 2) treten vornehmlich im Erwachsenenalter auf und machen etwa $60 \%$ aus [5].

Die klassischen Nahrungsmittelallergene, wie Hühnereiweiß und Milcheiweiß, sind eher im Kindesalter Ursache einer Nahrungsmittelallergie und gehen dann zurück, während im Erwachsenenalter Nahrungsmittelallergien und damit einhergehende schwere Reaktionen, z. B. Anaphylaxien durch pollenassoziierte NahrungsmittelallergenQuellen, wie Sellerie, Haselnuss oder Soja, auftreten.

Nahrungsmittel stehen an erster Stelle als Ursache für die Auslösung schwerer allergischer Reaktionen bei Kindern. Die häufigsten Anaphylaxieauslöser sind hier Erdnüsse und andere Leguminosen, Baumnüsse, vor allem die Haselnuss, tierische Produkte wie Hühnereiweiß und Milcheiweiß, aber auch Gewürze, Gemüse und Früchte können schwere allergische Reaktionen auslösen. Bei Erwachsenen sind es vor allem Mollusken und Krustentiere, aber auch Fisch, Hühnerei und selten Schweinefleisch, dann erst gefolgt von Gemüse und Früchten, aber auch Erdnüssen und Baumnüssen [6]. Im Erwachsenenalter können auch schwere Reaktionen, wie Anaphylaxien durch pollenassoziierte Nahrungsmittelallergen-Quellen, wie Sellerie, Haselnuss oder Soja, auftreten. 
Häufige Anaphylaxieauslöser unter den Nahrungsmitteln sind Ei, Milch, Fisch, Schalentiere, Erdnüsse, Lupinensamen, Soja und Baumnüsse.

\section{Einteilung der Nahrungsmittelallergien nach Sensibilisierungsweg \\ $\nabla$}

1998 wurde eine Klassifikation der IgE-vermittelten Nahrungsmittelallergien nach Sensibilisierungsweg vorgeschlagen, welche die altersabhängige unterschiedliche Häufigkeit des Auftretens und des Allergenspektrums sowie die unterschiedliche Thermo- und Säurestabilität der entsprechenden Nahrungsmittelallergene berücksichtigt [7]. Dabei werden der natürliche Verlauf, die Toleranzentwicklung und die starke Altersabhängigkeit der Nahrungsmittelallergien sowie die molekulare Charakterisierung der wichtigen Allergene und entsprechender Kreuzreaktionen einbezogen.

\section{Nahrungsmittelallergie vom Typ A:}

- Auftreten im Säuglings- oder Kleinkindesalter

- Sensibilisierung über den Gastrointestinaltrakt mit relativ stabilen Allergenen

- wichtigste Allergene: Milch, Ei, Fisch und Erdnuss

- Rückbildung mit Reifung der oralen Toleranz (Für die Erdnussallergie besteht allerdings die Gefahr der Persistenz bis in das Erwachsenenalter hinein.) [7]

\section{Nahrungsmittelallergie vom Typ B:}

- betroffen: ältere Kinder, Jugendliche und Erwachsene

- Sensibilisierung über den Respirationstrakt

- Aeroallergene (Pollen, Latex) induzieren die Bildung allergenspezifischer IgE-Antikörper, die Kreuzreaktionen mit ähnlichen Proteinen in pflanzlichen Nahrungsmitteln (Obst, Gemüse, Nüsse) aufweisen.

- Art und Lokalisation der klinischen Symptome werden durch die Stabilität der kreuzreagierenden Nahrungsmittelallergene bestimmt, wobei die meisten Patienten nur milde Symptome aufweisen.

- in Mitteleuropa die häufigste Form von Nahrungsmittelallergien [7]

\section{Nahrungsmittelallergie vom Typ C:}

- betroffen sind meistens erwachsene Frauen

- häufig liegt keine atopische Disposition vor und die Sensibilisierung erfolgt im Gastrointestinaltrakt gegen wenige, relativ verdauungsstabile Proteine

- Dieser Typ der Nahrungsmittelallergie ist selten [7].
Nahrungsmittelallergie vom Typ D:

- von B. Wüthrich postulierter 4. Nahrungsmittelallergietyp, bei welchem die Sensibilisierung primär perkutan stattfindet [8].

\section{Augmentationsfaktoren}

$\nabla$

Zu den Augmentationsfaktoren zählen individuelle, z. B. Alkohol, körperliche Belastung und Medikamente, hierunter insbesondere die nicht steroidalen Antiphlogistika [9] sowie allgemeine Augmentationsfaktoren wie Pollensaison.

\section{Risikofaktoren}

\section{$\nabla$}

Patienten mit Erkrankungen aus dem atopischen Formenkreis, wie atopisches Ekzem, allergische Rhinokonjunktivitis und allergisches Asthma, sind besonders gefährdet, eine Anaphylaxie auf ein Nahrungsmittel zu entwickeln.

Ein bedeutender Risikofaktor schwerer oder tödlich verlaufender Nahrungsmittelallergien ist vorbestehendes Asthma bronchiale, die häufigste Todesursache ist der Status asthmaticus. Zumeist war in diesen Fällen die Allergie bekannt und durch eine milde Symptomatik gekennzeichnet. Häufige Auslöser sind Ei, Milch, Fisch, Schalentiere, Erdnüsse, Lupinensamen und Baumnüsse [1].

Risikofaktoren sind Erkrankungen aus dem atopischen Formenkreis, wie atopisches Ekzem, allergische Rhinokonjunktivitis und allergisches Asthma. Patienten mit Asthma bronchiale sind besonders gefährdet, eine Anaphylaxie auf ein Nahrungsmittel zu entwickeln.

Die besonders gefährdete Altersgruppe liegt zwischen 12 und 20 Jahren. Dabei kommt es häufig zu einem versehentlichen Genuss der bekannten Allergene [1].

Die Schwere der vorangegangenen klinischen Reaktion ist kein verlässlicher prognostischer Hinweis für die Schwere einer Folgereaktion [1].

Die Aufklärung ist essenziell, da gerade junge Patienten in der Adoleszenz die Verträglichkeit der ihnen bekannten Nahrungsmittelallergene hin und wieder unkontrolliert ausprobieren [1]. Eine Diätberatung wird auch die Labilität/Stabilität der Nahrungsmittelallergene einbeziehen müssen. Insbesondere bei den pollenassoziierten Nahrungsmittelallergenen handelt es sich zumeist (!) um hitze-, thermo- und säurelabile Allergene, Ausnahmen sind das Gly m 4 aus der Sojabohne und das Lipidtransferprotein aus dem Pfirsich. Das bedeutet, dass z. B. birkenpollenallergische Patienten Kern- und Steinobst in ausreichend lange gekochter und gegarter Form ohne Beschwerden

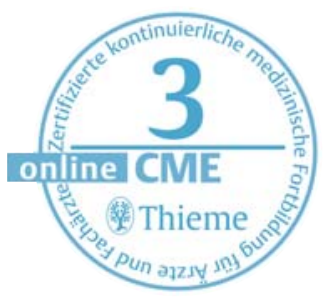


Tab. 1 Übersicht der Bet-v-1-homologen Proteine in Nahrungsmitteln.

\begin{tabular}{|c|c|c|}
\hline Nahrungsmittel & Spezies & Bet-v-1-homologes Allergen \\
\hline goldene Kiwi & Actinidia chinensis & Act $c 8$ \\
\hline grüne Kiwi & Actinidia deliciosa & Act $d 8$ \\
\hline Sellerie & Apium graveolens & Apig $1^{*}$ \\
\hline Erdnuss & Arachis hypogaea & $\operatorname{Arah} 8^{*}$ \\
\hline Haselnuss & Corylus avellana & Cora $1^{*}$ \\
\hline Karotte & Daucus carota & Dau c 1 \\
\hline Erdbeere & Fragaria ananassa & Fra a 1 \\
\hline Walderdbeere & Fragaria vesca & Fra v 1 \\
\hline Sojabohne & Glycine maxima & Gly m 4* \\
\hline Tomate & Lycopersicon esculentum & Lyc e 4 \\
\hline Apfel & Malus domestica & Mal d $1^{*}$ \\
\hline Aprikose & Prunus armeniaca & Pruar $1^{*}$ \\
\hline Süßkirsche & Prunus avium & Pruav $1^{*}$ \\
\hline Pfirsich & Prunus persica & Prup $1^{*}$ \\
\hline Birne & Pyrus communis & Pyr c 1 \\
\hline Himbeere & Rubus idaeus & Rub i 1 \\
\hline Mungobohne & Vigna radiata & Vigr 1 \\
\hline Kartoffel & Solanum tuberosum & pSTH-2 und -21 \\
\hline Petersilie & Petroselinum crispum & pcPR1 und 2 \\
\hline
\end{tabular}

essen können, da die verantwortlichen Allergene durch Erhitzen z.T. zerstört werden. Eine absolute Sicherheit gibt es hingegen nicht.

Ein weiteres Beispiel ist die Sellerieallergie:

Patienten mit einer birkenpollenassoziierten Sellerieallergie reagieren auf die eher hitzelabilen Komponenten, Patienten mit einer beifußassoziierten Sellerieallergie hingegen auf die hitzestabilen Epitope [1]

In Zukunft wird die Einzelallergenanalyse eine bessere Prognostizierbarkeit klinisch relevanter Kreuzreaktionen möglich machen.

\section{Kreuzreaktionen und ihre Bedeutung für Nahrungsmittelallergien}

Unter einer Kreuzreaktion versteht man die Bindung von Antikörpern an bzw. die Aktivierung spezifisch sensibilisierter T-Lymphozyten mit unterschiedlichen Substanzen (Proteine, Kohlenhydrate, Glykoproteine), die identische oder ähnliche antigene Determinanten aufweisen, was zum einen auf einer hochgradigen Sequenzähnlichkeit (lineare Epitope), zum anderen auf der Ähnlichkeit der 3-D-Struktur (Konformation, Konformationsepitope) beruhen kann.

Birkenpollenallergiker reagieren häufig auf den Genuss von Baumnüssen, Kern-/Steinobst, Karotte und Soja, Beifußallergiker häufig auf Sellerie und Kräuter. Aber auch Latexallergiker können auf pflanzliche Nahrungsmittelallergen-Quellen reagieren (naturlatexassoziiertes Fruchtallergiesyndrom). Hier sind insbesondere Banane, Kiwi, Avocado, Esskastanie, Ananas, Tomate, Paprika und seit 2002 auch die südamerikanische Acerola-Kirsche zu nennen [10]. Gräser- und Getreideallergiker können auf Mehle und Tomate reagieren, Hausstaubmilbenallergiker auf Krusten-, Schalen- und Weichtiere sowie Tierepithelienallergiker auf Fleisch [1, modifiziert]. Immunologische Kreuzreaktionen sind bei Jugendlichen und Erwachsenen die häufigste Ursache für Nahrungsmittelallergien. Bei Nahrungsmittelallergien durch Kreuzreaktionen kann bereits der Erstkontakt mit dem Nahrungsmittel zu allergischen Symptomen führen.

Bei der Aufklärung der für die Kreuzreaktion verantwortlichen Epitope (sequenzielle oder konformationelle Epitope) ist die Einteilung in Proteinfamilien hilfreich [11]. Die Einteilung in bestimmte Proteinfamilien erfolgt dabei anhand der Struktur, Funktion und biologischen Aktivität.

\section{Allergenfamilien}

Die wichtigsten Proteinfamilien, in welche sich die pflanzlichen Nahrungsmittelallergene eingruppieren lassen, sind Lipidtransferproteine (LTP), Profiline, die Bet-v-1-Superfamilie, die Cupine [11] und die kreuzreaktiven Kohlenhydratepitope (cross-reactive carbohydrate determinants: CCD).

Die offizielle Allergen-Nomenklatur benutzt für Einzelallergene den lateinischen Namen der Allergenquelle abgekürzt und eine Nummer in der Reihenfolge der Entdeckung, z. B. heißt das Majorallergen der Birke Betula verrucosa Bet v 1 [12]

\section{Bet-v-1-Superfamilie}

Die bei Jugendlichen und Erwachsenen am häufigsten anzutreffende pollenassoziierte Nahrungsmittelallergie basiert auf einer Kreuzreaktion zwischen Inhalations- und Nahrungsmittelallergenen. Besonders häufig ist dieses Phänomen bei Patienten mit Birkenpollenallergie [13], wofür das Bet v 1 (das Majorallergen der Birke) über seine entsprechenden homologen Moleküle in den pflanzlichen Nahrungsmitteln verantwortlich ist ( Tab. 1).

Bet v 1 ist als ein Vertreter der weit verbreiteten pflanzlichen pathogenesis-related Proteins bekannt. Die Aufklärung der molekularen Struktur des Bet $\mathrm{v} 1$ erlaubte die Identifikation strukturverwandter Proteine in anderen Allergenquellen und somit die Erfassung einer Superfamilie, innerhalb der die Bet-v-1-Familie nur eine darstellt [11] In den nordeuropäischen Gegenden mit starkem Birkenbewuchs ist das Phänomen solcher Kreuzreaktionen sehr häufig und klinisch zumeist milde, d.h. im Sinne eines oralen Allergiesyndroms verlaufend. Eine Ausnahme hiervon ist das Bet-v-1-homologe Protein der Sojabohne (Glycine maxima, Gly $\mathrm{m} 4$ ), welches mit schweren allergischen Symptomen bei Birkenpollenallergikern nach Soja-Genuss assoziiert ist [14].

\section{Profiline}

Profiline sind konservierte Proteine mit einem Molekulargewicht zwischen 12 und $15 \mathrm{kDa}$ und finden sich in allen eukaryotischen Zellen. Gegen 
Profiline sind ca. 20\% der Patienten, die von einer Pollenallergie betroffen sind, sensibilisiert. Die entsprechenden IgE-Antikörper sind zwischen Pollen- und Nahrungsmittelprofilinen hoch kreuzreaktiv. Sie scheinen für Nahrungsmittelallergien allerdings klinisch häufig nicht relevant zu sein [11].

\section{Kreuzreaktive Kohlenhydratdeterminanten (CCD)}

Allergenspezifische IgE-Antikörper erkennen und binden nicht nur Peptid-Epitope, sondern auch Epitope von N-Glykanen, sogenannte Kohlenhydratepitope. Da sie für viele verschiedene Kreuzreaktionen verantwortlich sind, werden sie auch kreuzreaktive Kohlenhydratepitope (-Determinanten) genannt. Da Pflanzenallergene zumeist Glykoproteine sind und sie gehäuft in Pollen, besonders Gräserpollen, sowie in pflanzlichen Nahrungsmitteln und Naturlatex vorkommen, findet man gegen CCD gerichtete IgE-Antikörper vornehmlich in Seren von Patienten mit multiplen Sensibilisierungen gegen pflanzliche Allergene. Bislang gibt es nur sehr wenig Daten zur Prävalenz von Anti-CCD-IgE bei Patienten mit Nahrungsmittelallergie: Bei 10-50\% derjenigen, die auf Sellerie, Karotte, Zucchini oder Tomate sensibilisiert waren, fanden sich Anti-CCD-IgE im Serum [46].

Escherichia coli (E. coli), die Bakterien, die zur Massenproduktion von rekombinanten Proteinen (Allergenen) verwendet werden, sind nicht in der Lage, Proteine mit posttranslationaler Modifikation, wie z.B. einer Glykosylierung, herzustellen. Daher weisen rekombinante Allergene, wie sie derzeit mehr und mehr in der allergologischen Labordiagnostik eingesetzt werden, keine CCD auf, wenn sie in E. coli exprimiert werden. Dieses muss für die Diagnostik gerade zur Aufklärung von Kreuzreaktionen berücksichtigt werden.

Palacin u. Mitarb. beschrieben z.B. die mögliche Assoziation zwischen respiratorischer Allergie auf Getreidemehle als Bäcker-Asthma und einer Kiwiallergie auf der Basis kreuzreaktiver Kohlenhydratdeterminanten und Thiolproteasen, die sie für einige Patienten als ursächlich für die WeizenKiwi-Kreuzreaktion nachwiesen [15].

\section{Prolamin-Superfamilie}

Die Prolamine sind eine Gruppe von Speicherproteinen, die vor allem in den Samen von Getreiden und anderen Mitgliedern der Gräserfamilie vorkommen. Mit den Prolaminen verwandte Proteine sind die 2S-Albumine und die nicht spezifischen LTP. Eine klinisch bedeutsame Allergie gegenüber Getreideprolaminen ist die Weizenallergie, wobei hier das Omega-5-Gliadin und das Glutenin eine besondere Bedeutung haben. Typische 2S-Albumine sind die der Paranuss. Einige der wichtigsten Baumnuss-, Erdnuss- und Samenallergene sind 2S-Albumine. So gehören die Erdnussallergene (Arachis hypogaea) Ara h 2 und
Ara h 6 zum Typ Conglutin der 2S-Albumine. Sie werden derzeit als die wichtigsten Erdnussallergene erachtet [11].

\section{Cupin-Superfamilie}

Zu dieser Familie gehören u.a. die Globulin-Speicherproteine, welche einen großen Anteil an der menschlichen Nahrung ausmachen. Am besten untersucht sind die Leguminosen, hierunter besonders die Erdnuss und die Sojabohne. Die Globuline werden unterteilt in 7S-Globuline (Viciline) und 11S-Globuline (Legumine). Bereits als Allergene bekannte Vertreter sind das Ara h 1 (das allergene Vicilin der Erdnuss), welches eines der Hauptspeicherproteine der Erdnuss ist und von über 90\% der Erdnussallergiker erkannt wird. Gly $\mathrm{m}$ 5.01, ebenfalls ein Vicilin, ist eines der wichtigsten Allergene der Sojabohne. Das Cor a 11 der Haselnuss gehört dazu. Auch die Legumine bestehen aus Proteinen, die bereits als Allergene bekannt sind, z. B. das Cor a 9 der Haselnuss oder das Ana o 2 der Cashewnuss [11].

\section{Versteckte Allergene}

$\nabla$

Ein verstecktes Allergen

1. ist ein allergieauslösendes Nahrungsmittel, das für den Verbraucher nicht erkennbar in einem Lebensmittel vorhanden ist.

2. kann Zutat eines Erzeugnisses sein oder aufgrund einer unbeabsichtigten Verunreinigung während der Herstellung in ein Produkt gelangen.

Seit 2003 besteht in der Europäischen Gemeinschaft eine Deklarationspflicht für die inzwischen folgenden 14 Nahrungsmittel gemäß Richtlinie 2003/89/EG zur Änderung der Richtlinie 2000/ 13/EG vom 25.11. 2003 und der Aktualisierung vom 22. 12. 2006:

Getreide, Hühnerei, Erdnuss, Baumnüsse, Soja, Senf, Krebstiere, Fisch, Kuhmilch, Sellerie, Sesam, Mollusken (Muscheln, Tintenfisch), Lupine und schwefeldioxid-/sulfithaltige Produkte [16].

\section{Neue Allergenquellen und Novel Food}

Novel Food setzt sich definitionsgemäß aus Lebensmitteln oder Lebensmittelzutaten zusammen, die neuartig (neu entdeckt oder neu entwickelt) sind, die normalerweise nicht in nennenswertem Umfang in Europa verzehrt werden bzw. nicht im Handel sind. (Anmerkung: Seit 2004 fallen gentechnisch veränderte Nahrungsmittel unter eine eigene gesetzliche Regelung und nicht mehr unter die Novel-Food-Verordnung [17].) Kiwi z.B. wurde als Novel Food in die europäische Diät eingeführt, und schon wenig später erfolgte die Erstbeschreibung der Kiwi-Allergie [18]. Die Lupine gehört zu den eiweißreichsten und fettreichsten Leguminosen und findet daher

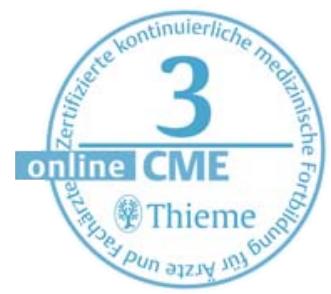


einen zunehmenden Einsatz in der Nahrungsmittelindustrie. Die Fettsäurezusammensetzung ist der des Sojaöls vergleichbar. Lupinenmehl wird seit den 1990er-Jahren als Nahrungsmittelzusatz in Europa verzehrt. Insgesamt sind seit Erstbeschreibung einer Allergie auf Lupine bei einem erdnussallergischen Kind im Jahr 1994 weltweit inzwischen ca. 155 Fälle einer Lupinenallergie mehr oder weniger gut dokumentiert [19]. Dabei treten die Symptome in der Regel bereits wenige Minuten nach dem Kontakt auf. Als mögliche Auslöser für Kreuzreaktionen zu anderen Nahrungsmitteln kommen Erdnuss, Sojabohne, Linse, grüne Bohne und Erbse infrage. Während bislang Kreuzallergien zwischen Lupine und Sojabohne bzw. Linse, Bohne und Erbse kaum eine Rolle spielen, scheint die Kreuzreagibilität zwischen Lupine und Erdnuss relativ häufig klinisch relevant zu sein. Seit 2006 ist die Lupinenallergie auch in Deutschland bekannt [20].

Ein weiteres aktuelles Beispiel neuer Nahrungsmittelallergen-Quellen ist der Granatapfel. Weltweit sind erst 12 Fälle publiziert [21].

\section{Diagnostik \\ $\nabla$}

Die Diagnostik der Nahrungsmittelallergie setzt sich zusammen aus Anamnese, unterstützt durch ein Symptom-Ernährungs-Tagebuch, sowie dem Nachweis allergenspezifischer IgE-Antikörper einschließlich Gesamt-IgE-Konzentration im Serum und In-vivo-Untersuchungen, bestehend aus Hauttests und oralen Provokationstestungen (offen oder doppelblind und placebokontrolliert) nach Eliminationsdiät [1]. Die Kartoffel-Reis-Diät ist nicht mehr üblich, sondern wird dort, wo es nötig ist, durch die oligoallergene Basisdiät ersetzt, die sich im Einzelfall aus individuell verträglichen, seltener allergieauslösenden Nahrungsmitteln zusammensetzt [22].

Die Anamnese einer Nahrungsmittelunverträglichkeit ist oft sehr komplex und schwierig. Dabei gilt es, klare Beziehungen zwischen der klinischen Symptomatik und der potenziell auslösenden Nahrungsmittelallergen-Quelle zu erfassen und des Weiteren zu differenzieren, ob es sich um eine Nahrungsmittelunverträglichkeit oder um eine immunologisch bedingte und somit „echte“ Nahrungsmittelallergie handelt. Davon hängen weitere diagnostische und therapeutische Maßnahmen entscheidend ab. Die höchste Wertigkeit hat die Anamnese bei nahrungsmittelallergiebedingten Sofortreaktionen, während es bei Spätreaktionen oft schwierig ist, die ursächlichen Zusammenhänge herzustellen [2].

Es ist dabei sehr wichtig, dass der untersuchende Arzt über weitreichende Kenntnisse zu potenziell auslösenden Allergenen und möglicherweise vorhandenen Kreuzallergien verfügt. Hierbei sind die folgenden Internetseiten sehr hilfreich, z. B. www. allergome.org, www.meduniwien.ac.at/allergens/ allfam, www.iuisonline.org.

Patienten mit Verdacht auf Nahrungsmittelunverträglichkeit sollten ein Symptom-ErnährungsTagebuch führen. Es ist eine wichtige Unterstützung der Anamneseerhebung, da es nicht nur potenziell zeitliche/ursächliche Zusammenhänge sichtbar machen kann, sondern auch einer allergologisch ausgebildeten Ernährungsfachkraft viele Informationen über die Lebens- und Ernährungsgewohnheiten der Patienten vermittelt. Dies gilt insbesondere für sehr komplexe Anamnesen [1].

\section{Allergologische In-vitro-Diagnostik}

Für die klinische Symptomatik ursächliche Nahrungsmittelallergene sind als solches oft nicht sofort erkennbar, was darauf beruhen kann, dass es sich um versteckte Allergene handelt. Des Weiteren können Symptome durch neue Allergene aus bestimmten, auf dem nationalen Markt neu eingeführten Nahrungsmitteln hervorgerufen worden sein. Teilweise stellen einige der Extrakte für allergologische Haut- und Labortests aufgrund ihrer Zusammensetzung das relevante Allergenspektrum nicht dar und sind daher für den Allergennachweis unzulänglich.

Ein wichtiges Instrument für die Allergie-Diagnostik ist die Detektion allergenspezifischer IgE-Antikörper, die eine klare und somit sichere Diagnose zum Ziel hat, sodass der Patient den Allergieauslöser gezielt meiden kann bzw., wenn das nicht möglich ist oder nicht ausreicht, die für den jeweiligen Patienten geeignete allergenspezifische Immuntherapie eingeleitet werden kann.

Als pflanzliche (z.B. Früchte, Gemüse, Nüsse) und tierische (z.B. Kuhmilch, Hühnerei, Fisch) Allergenquellen werden Extrakte verwendet, wenn sie stabil genug sind.

Der Nachweis von spezifischen IgE-Antikörpern gegen Hühnerei, Kuhmilch, Erdnuss oder Fisch in hohen Konzentrationen ist mit erhöhtem Allergie-Risiko verknüpft. Dennoch kann nur selten auf eine bestätigende orale Provokation verzichtet werden [23].

Die Identifizierung und Charakterisierung relevanter Einzelallergene, die Klonierung und Produktion als rekombinante allergene Proteine sowie die Synthese IgE-Epitop-darstellender Peptide hat eine komponentenaufgelöste Diagnostik (component-resolved diagnosis) dadurch möglich gemacht, dass nun IgE-Antikörper, die für ein allergenes Protein oder ein sequenzielles Epitop spezifisch sind, nachgewiesen werden können. So lassen sich individuelle Sensibilisierungsmuster der Patienten gegen verschiedene Proteine, z. B. allergene Nahrungsmittel, Pollen etc. oder homologe Proteine in verschiedenen Nahrungsmitteln $(\bullet$ Tab. 1), oder verschiedene Epitope eines einzelnen Allergenmoleküls erstellen. 
Es gibt neuerdings für NahrungsmittelallergenQuellen pflanzlichen Ursprungs einige Markerallergene [23,24], während die Einzelallergene im Falle der Diagnostik einer Allergie auf tierische (Glyko-)Proteine derzeit noch eine untergeordnete Rolle spielen.

Als Risikomoleküle für systemische Reaktionen gelten gegenwärtig - durchaus abhängig von geografischen Faktoren - folgende Einzelallergene: das Erdnuss-Speicherprotein Ara h 2 (Arachis hypogaea), das Lipidtransferprotein (LTP) der Erdnuss (Ara h 9), das Haselnuss-LTP Cor a 8 (Corylus avellana), das Pfirsich-LTP Pru p 3 (Prunus persica) und das Weizenprotein Omega-5-Gliadin Tri a 19 (Triticum aestivum) [24]

Der Nachweis von IgE-Antikörpern gegen Einzelallergene stellt eine molekülspezifische Diagnostik dar, deren Aussagefähigkeit je nach Allergenquelle und klinischer Charakterisierung der Einzelallergene zu klären ist.

Zelluläre Verfahren zum indirekten Nachweis einer IgE-mediierten Reaktion (Histamin-Freisetzungstest, Basophilen-Aktivierungs- oder Degranulationstest, Leukotrien-Freisetzungstest [Cellular Antigen Stimulation Test: CAST] sowie der Lymphozyten-Stimulationstest) sind methodisch aufwendig und gelten noch immer als ergänzende Verfahren im Rahmen von Einzelfallanalysen und wissenschaftlichen Fragestellungen.

Untaugliche Verfahren im Rahmen der Nahrungsmittelallergie-Diagnostik sind Bioresonanz, Kinesiologie, Elektroakupunktur, zytotoxischer Lebensmitteltest (Methoden ohne Aussagekraft und/oder Überprüfung), Lymphozyten-Transformationstest, nahrungsmittelspezifisches $\operatorname{IgG}$ und IgG4 (Methoden mit unzulässiger Interpretation) $[25,26]$.

\section{In-vivo-Allergietestungen \\ Hauttestungen}

Ebenso wie der allergenspezifische IgE-Nachweis im Serum der Patienten zählt auch der Hauttest zu den Methoden, mithilfe derer lediglich eine spezifische Sensibilisierung, keinesfalls aber die Allergie (klinisch relevante Sensibilisierung) nachgewiesen werden kann. Zum Nachweis der Soforttypallergien ist hier der Prick-Test die am häufigsten verwendete Variante und dient als Basistest (resümiert in [27]). Allerdings sind die zur Verfügung stehenden, kommerziell erhältlichen Nahrungsmittelallergen-Extrakte für die Hauttestung oft labil. Im Gegensatz zu den Extrakten der bedeutsamsten Inhalationsallergene gibt es bislang für die Nahrungsmittel keine vergleichbar gut standardisierten Testlösungen für alle relevanten Allergenquellen. Während das Risiko von systemischen allergischen Reaktionen nach Prick-Test mit kommerziell erhältlichen Nahrungsmittel-Allergenextrakten eher als gering einzuschätzen ist, stellt sich das für die Testung mit rohem Material (Prick-zu-Prick-Testung) ganz anders dar.
Da nichts über den Allergengehalt roh getesteter Nahrungsmittel bekannt ist - da dieser u.a. abhängig ist von Spezies und Reifungsgrad des Obstes und des Gemüses bzw. von der Art der Zubereitung -, kann es hier durchaus zu systemischen Reaktionen kommen.

Beim Prick-zu-Prick-Test geht der Untersucher zuerst mit der Pricklanzette in das zu testende Nahrungsmittel, bevor er anschließend in die Haut der Testperson sticht.

Wichtig ist zudem, dass für diese Nativtestung nur frisches Obst und Gemüse und nicht tiefgefrorene Ware verwendet wird, da beim Auftauprozess Allergenstrukturen zerstört werden können. Rohmaterial ist unterschiedlich gut für den Prickzu-Prick-Test geeignet [28]. Aufgrund ihres irritativen Potenzials bedingt geeignet sind: Ananas, Erdbeeren, Gewürze, Kiwi, Senf und Tomate [28]. Eine eingeschränkte Bedeutung für die Diagnostik der Nahrungsmittelallergie haben aufgrund der fehlenden klinischen Reproduzierbarkeit der Reibtest und der Scratch- bzw. Skarifikationstest, der zudem mit Traumatisierung der Haut und möglicherweise falsch positiven Ergebnissen einhergehen kann [29]. Auch der Intrakutantest spielt für die Diagnostik der Nahrungsmittelallergie eine untergeordnete Rolle. Er ist aufgrund vielfach erhöhter Sensitivität häufiger unspezifisch positiv. Darüber hinaus birgt er ein höheres Risiko allergischer Systemreaktionen [29]. Eine Ausnahme zur Verwendung des Skarifikationstests stellt der Scratch-Chamber-Test zur Diagnostik einer Proteinkontaktdermatitis dar.

Der Atopie-Patch-Test, insbesondere mit Kuhmilch und Hühnerei, kann zur Diagnostik einer durch den Genuss der entsprechenden Nahrungsmittel verschlechterten atopischen Dermatitis eingesetzt werden. Im Falle der Positivität sollte die weitergehende Diagnostik in einer gezielten diagnostischen Eliminationsdiät, ggf. gefolgt durch eine orale Provokation, durchgeführt werden, bevor eine Diät empfohlen wird [1].

\section{Eliminationsdiät und Provokationstestung}

Der spezifische Verdacht gegen ein Nahrungsmittel als Auslöser der Symptomatik kann durch eine mindestens 7-tägige Eliminationsdiät erhärtet werden, was der Patient nach einer entsprechenden Diätberatung ambulant zu Hause durchführen kann. Ist die Anamnese allerdings sehr viel komplexer, vermag die Eliminationsdiät nur begrenzt weiterzuhelfen. Hier müssen dann in der Regel im Anschluss an die diagnostische Eliminationsdiät orale Provokationstests durchgeführt werden, wobei im Kindesalter zumeist noch die offene orale Provokation, im Erwachsenenalter allerdings vorzugsweise die doppelblind-placebokontrollierte Provokationstestung durchgeführt werden sollte. Hier ist die Zusammenarbeit mit allergologisch geschulten Ernährungsfachkräften sehr wichtig $[1,22]$.

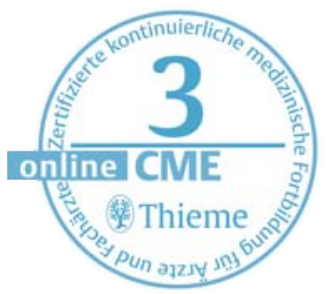




\section{Doppelblind-placebokontrollierte} Provokationstestung

Unter den Provokationstests gilt die doppelblinde, placebokontrolliert durchgeführte orale Nahrungsmittelprovokation als der Goldstandard der NahrungsmittelallergieDiagnostik. Orale Provokationstests sollten generell in der symptomarmen und möglichst stabilen Phase der Erkrankung bzw. bei Erscheinungsfreiheit durchgeführt werden.

Neben der standardisiert durchgeführten Nahrungsmittelallergie-Diagnostik erlaubt die orale doppelblind-placebokontrollierte Provokation zudem die Bestimmung der Schwellenwerte, welche die Proteinkonzentration darstellen, ab der es zur Entwicklung von Symptomen kommt. Dieser Parameter ist ein Wert, der für die Deklaration einzelner Nahrungsmittel in komplex zusammengesetzten Speisen eine Bedeutung hat, da es dem Verbraucher in Zukunft eine bessere Übersicht darüber verschafft, welche Nahrungsmittel er zu sich nehmen kann und welche nicht. Der Warnhinweis vieler Hersteller „Kann Spuren von ... enthalten“ ist nicht verpflichtend und erfolgt aus Gründen der Produkthaftung. Er verunsichert aber den Verbraucher, da er letztendlich bezüglich allergieauslösender Konzentrationen möglicherweise infolge von Kontamination mit potenziellen Allergenen nicht aussagekräftig ist.

Die Verblindung der zu testenden Nahrungsmittel ist insbesondere bei solchen Nahrungsmitteln, die mit starkem Eigengeschmack einhergehen, oft sehr schwierig. Auch hier ist die Unterstützung durch eine allergologisch geschulte Ernährungsfachkraft essenziell.

\section{Offene orale Provokationstestung}

Die offene orale Provokationstestung mit Nahrungsmitteln hat dann eine starke diagnostische Aussagekraft, wenn das Ergebnis negativ ausfällt [1].

Problematisch wird die Interpretation bei fehlender Objektivierbarkeit und bei zu erwartenden Spätreaktionen [1].

Systemische Antihistaminika sollten mindestens 72 Std. zuvor abgesetzt worden sein. Im Falle der Diagnostik bei möglicherweise nahrungsmittelgetriggerter atopischer Dermatitis sollte ein schwaches Glukokortikoid maximal einmal täglich topisch eingesetzt werden. Ansonsten sollte auf Steroide, systemisch und extern, verzichtet werden. Je nach Ausprägung des Hautzustands sollte man vor Beginn der Provokation die Lokaltherapie intensivieren, um sie dann gleichmäßig fortzusetzen [2].

Die Applikation von Allergenen in Kapselform ist ungeeignet, da nur eine begrenzte Menge von Nahrungsmitteln auf diese Weise verabreicht werden kann und eine mukokutane Reaktion, wie das orale Allergiesyndrom, bei dieser Anwendung gar nicht auftreten kann. Zudem ist diese
Form der Provokationstestung für Säuglinge und Kleinkinder unmöglich [1].

Orale Provokationstestungen sollten mit sicherem intravenösem Zugang und unter schrittweiser Dosissteigerung (Titration) durchgeführt werden. Besondere Risikofaktoren sind hochpotente Allergene (Erdnüsse), vorbestehendes Asthma bronchiale und lange Karenz (d.h. langes Zeitintervall zwischen letztem Genuss des Nahrungsmittels und Reexposition). Orale Provokationstestungen können ambulant durchgeführt werden. Indikationen für eine stationäre Testung bestehen allerdings bei bedrohlichen Reaktionen in der Vorgeschichte und zusätzlich unklarem Zusammenhang zwischen Reaktion und der entsprechenden Nahrungsallergenquelle sowie bei Auftreten von Symptomen außerhalb der ambulanten Beobachtungszeit und für das Säuglings- und Kleinkindalter [1].

Die Testergebnisse beurteilt ein Arzt. Generell ist zwischen subjektiven und objektiven Symptomen zu unterscheiden. Im positiven Fall muss die ergänzende Diagnostik durch Detektion allergenspezifischer IgE-Antikörper im Serum und Hauttests zur Differenzierung, ob es sich um eine Nahrungsmittelunverträglichkeit oder um eine immunologisch bedingte Nahrungsmittelallergie handelt, herangezogen werden. Negative Ergebnisse einer Provokationstestung können falsch negativ sein, sofern zuvor bei dem Patienten Augmentationsfaktoren (z.B. im Fall der anstrengungsinduzierten Weizen-Anaphylaxie) oder zusätzlich der Genuss von Kaffee, Alkohol und nicht steroidalen Antiphlogistika bestanden [1].

Sofern diese also bekannt sind, sollten Augmentationsfaktoren Teil der Provokationstestung sein. Zudem ist denkbar, dass auch Kombinationen mehrerer Nahrungsmittel erst die allergische Reaktion auslösen [1].

Oft kann nur mittels Provokationstestung die klinische Aktualität und Bedeutung nachgewiesener Sensibilisierungen gegen Nahrungsmittel gesichert werden. In den Fällen allerdings, in denen die Anamnese insbesondere bei schweren allergischen Reaktionen auf ein Nahrungsmittel im Bezug auf den Auslöser eindeutige Hinweise gibt, wird eine bestätigende Provokationstestung als nicht sinnvoll und unnötig gefährlich erachtet $[1,22]$.

Bedrohliche Soforttypreaktionen in der Vorgeschichte stellen eine Kontraindikation für die Durchführung von oralen Provokationstestungen mit dem entsprechenden Nahrungsmittel dar [22].

Eine Besonderheit ist die Provokationstestung bei möglicherweise durch Nahrungsmittel getriggerter atopischer Dermatitis. Etwa jedes 3. Kind im Alter unter 2 Jahren, welches an einer mittelgradigen oder schweren Dermatitis atopica erkrankt ist, reagiert auf ein Nahrungsmittel, die Hälfte der auf Nahrungsmittel reagierenden Kinder erleidet 
auch eine Verschlechterung des Ekzems. Das macht es notwendig, nach einer oralen Provokationstestung zu späteren Zeitpunkten (mindestens 16-24 Std.) die Haut durch den Arzt nochmals begutachten zu lassen. Hier spielt zudem die Testung mit Placebo eine wichtige Rolle. Diese sollte unter identischen Bedingungen durchgeführt werden [30].

Aufgrund der Dynamik gilt ebenfalls für das hämatogene Kontaktekzem die Notwendigkeit, zu späteren Zeitpunkten nach Provokationstestung den Hautbefund zu beurteilen (meist innerhalb eines Tages nach Allergenzufuhr). Als Auslöser sind hier Metallsalze, insbesondere Nickel, sowie pflanzliche Stoffe in Nahrungsmitteln, insbesondere Perubalsam, beschrieben [31].

Die Indikation zur Provokationstestung ergibt sich aus der Chronizität und Schwere des Krankheitsbilds und einem deutlich positiven Epikutantest. Auch hier sollte die zeitlich begrenzte Eliminationsdiät Teil der Stufendiagnostik sein, in diesem Fall allerdings über 4 Wochen durchzuführen [1].

\section{Differenzialdiagnose \\ $\nabla$}

Bei der Differenzialdiagnose sind pseudoallergische Reaktionen (Auslöser: biogene Amine, Additiva) auszuschließen. Da es hier nicht zu einer immunologisch (IgE-Antikörper-) vermittelten klinischen Reaktion kommt und es keine Sensibilisierungsphase gibt, spricht man von einer Pseudoallergie, die klinisch vergleichbar der immunologischen Soforttypreaktion abläuft.

Auch Stoffwechselkrankheiten (Enzymmangelzustände) sowie Intoxikationen, z.B. durch bakterielle Toxine, die mit Nahrungsmitteln in den menschlichen Organismus gelangen, zählen hierzu. Darüber hinaus sind Aversion und Projektion wichtige Differenzialdiagnosen [1].

\section{Therapie}

$\nabla$

Erstes Gebot ist immer, das relevante Allergen, sobald es ermittelt wurde, zu meiden (Allergenkarenz). Bei baumpollenassoziierten Nahrungsmittelallergien sind die relevanten Allergene im Stein- und Kernobst, welche vorrangig oropharyngeale Symptome hervorrufen, zumeist hitzelabil, sodass sie durch ausreichend langes Erhitzen zerstört werden und der Allergiker diese Nahrungsmittel so zubereitet genießen kann [32]. Die Allergenkarenz wird allerdings dort erschwert, wo Nahrungsmittelallergene als versteckte Allergene vorkommen können, oder dort, wo der Patient möglicherweise aufgrund von Kreuzreaktionen, deren Komplexität nicht bekannt ist, auf ihm unbekannte Nahrungsmittel schwere Reaktionen aufweist (z.B. Lupinenallergie bei Erdnussallergikern) [19]. Insbesondere im
Falle schwerer (anaphylaktischer) Reaktionen auf Nahrungsmittelallergene sollte die Karenz mit der Verordnung eines Notfallsets (schnell absorbierbares orales Antihistaminikum, Glukokortikosteroid und Adrenalin) kombiniert werden.

Die Diagnose von Nahrungsmittelallergien im Kindesalter, durch Provokationstests bewiesen, sollte nach Ablauf von 1 - 2 Jahren mittels Reexposition unter ärztlicher Aufsicht und abhängig vom Schweregrad unter stationären Bedingungen reevaluiert werden, da sich in der Zwischenzeit eine natürliche Toleranz entwickelt haben könnte [33-35].

Nach 1 - 2 Jahren Allergenkarenz im frühen Kindesalter sollte die entsprechende Diätempfehlung - je nach Schwere der Symptome ggf. stationär - per expositionem reevaluiert werden.

Das gilt nicht für Allergien gegen Erdnuss, Baumnüsse und Fisch, die eine zumeist lebenslange Persistenz zeigen [33]. Durch bewiesene Nahrungsmittelallergien notwendig gewordene Diäten sollten in Zusammenarbeit mit allergologisch ausgebildeten Ernährungsfachkräften zusammengestellt werden, damit es nicht zu einer Fehlernährung insbesondere im Kindesalter kommt [1].

\section{Medikamentöse Therapie der Nahrungs- mittelallergie}

Die therapeutische Intervention sollte erst erfolgen, nachdem die klinische Relevanz einer Nahrungsmittelallergie eindeutig nachgewiesen ist. Die Therapie der Nahrungsmittelallergie ist formal zu unterscheiden in Notfallmanagement [36], Selbstmedikation des Patienten und Präventivmaßnahmen.

Als Indikation für einen Adrenalin-Autoinjektor bei Nahrungsmittelallergien gelten die Vorgeschichte früherer schwerer Reaktionen, zunehmende Schwere der allergischen Symptome, Patienten mit systemischer Reaktion auf Nahrungsmittel und persistierendem - schlecht eingestelltem - Asthma bronchiale, systemische Allergie auf Erdnüsse, Baumnüsse und Sesam, Patienten, die auf geringste Mengen des Allergens reagieren, und Patienten mit Mastozytose [37].

Ein Notfallset besteht aus einem Adrenalin-Autoinjektor, einem Antihistaminikum und einem Glukokortikosteroid sowie bei einer Mitbeteiligung der unteren Atemwege zusätzlich aus einem inhalativen Beta-2-Agonisten [38,39]. Es sind allerdings bei der Verordnung des Adrenalins immer mögliche Kontraindikationen zu berücksichtigen. Sehr wichtig ist in diesem Zusammenhang die praktische Einweisung in den Gebrauch des Notfallsets, insbesondere der kommerziell erhältlichen Injektoren.

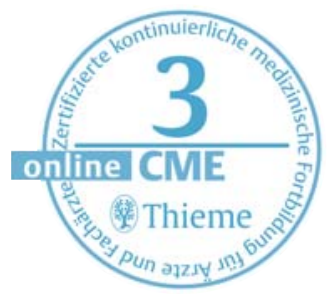


Spezifische Immuntherapie mit Nahrungsmittelallergenen

Subkutane und sublinguale spezifische Immuntherapie

Es gibt einzelne Studien, in denen untersucht wurde, ob eine allergenspezifische Immuntherapie gegen Pollen auch die entsprechend assoziierte Nahrungsmittelallergie positiv beeinflussen kann. Die Ergebnisse sind von Studie zu Studie sehr unterschiedlich, liegen aber insgesamt zwischen 30-84\% Erfolgsrate, wobei die wenigsten Studien placebokontrolliert waren. Als einer der wichtigsten Gründe dafür wird die Allergenmenge diskutiert, die möglicherweise im Sinne der pollenassoziierten Nahrungsmittelallergie suboptimal ist [37].

Daher sollte eine allergenspezifische Immuntherapie bei pollenassoziierter Nahrungsmittelallergie mit Pollenextrakten nur dann erfolgen, wenn eindeutige pollenbedingte Atemwegssymptome bestehen [37]

Ein weiteres Problem stellt die eingeschränkte Sicherheit entsprechender untersuchter Therapeutika dar, insbesondere bei den klassischen, systemischen Nahrungsmittelallergien, z.B. der Haselnuss- und Erdnussallergie. Die subkutane allergenspezifische Immuntherapie mit Nahrungsmitteln wurde bislang bei Erdnussallergikern untersucht, ging allerdings mit einer hohen Rate an Nebenwirkungen einher $[40,41]$. Eine sublinguale Immuntherapiestudie mit Nahrungsmitteln wurde bei Patienten mit systemischen Reaktionen auf Haselnuss durchgeführt [42].

\section{Spezifische orale Toleranzinduktion}

Im Rahmen einer spezifischen oralen Toleranzinduktion (SOTI) werden native Nahrungsmittel täglich in steigender Dosis oral verabreicht. Die Wirksamkeit dieser Methode wurde zwar mehrfach bei Patienten mit Kuhmilch- und Hühnereiallergie gezeigt $[43,44]$, aber der Effekt scheint leider nicht anhaltend zu sein. Dieses Verfahren wird insbesondere dort angewandt, wo Nahrungsmittel, wie z.B. Erdnüsse, nicht sicher gemieden werden können. Auch hier gibt es ein hohes Nebenwirkungspotenzial mit Reaktionen, die leicht bis mittelschwer verlaufen. Zudem muss das Nahrungsmittel nach Induktion der Toleranz regelmäßig weiter eingenommen werden, um diese Toleranz aufrecht zu erhalten. Dieses Verfahren wird derzeit außerhalb von kontrollierten Studien noch nicht empfohlen [37].

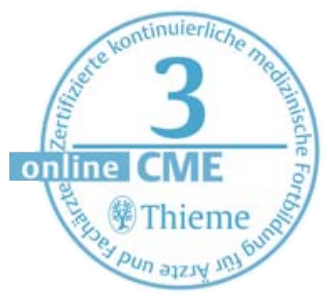

\section{Zusätzliche neue Therapieoptionen}

Der Einsatz von Anti-IgE-Antikörpern konnte im Off-Label-Use schon erfolgreich eine Anhebung der Reaktionsschwelle insbesondere bei Erdnussallergie induzieren [45].

\section{Kritisch zu betrachtende Therapieoptionen}

Die Arbeitsgruppe Nahrungsmittelallergie der DGAKI führt in ihrer Leitlinie [37] die folgenden Therapieformen als hinsichtlich der Effektivität nicht gesichert und daher als abzulehnende Verfahren auf: Rotationskost, Diäten aufgrund unsinniger IgG-Bestimmungen der Nahrungsmittelallergie, bioenergetische Verfahren wie Elektroakupunktur nach Voll- oder Bioresonanz.

Generell gilt, dass eine Fehlernährung durch einseitige Diäten unbedingt vermieden werden muss [37].

\section{Zusammenfassung}

$\nabla$

Nahrungsmittelallergien können sehr schwer verlaufen. Es gibt im Wesentlichen 2 Einteilungen der Nahrungsmittelallergene: in solche, die bereits im Kindesalter schwere systemische Reaktionen auslösen können (Klasse 1), und die pollenassoziierten Nahrungsmittelallergene, die vorwiegend im Erwachsenenalter relevant sind (Klasse 2). Eine weitere Einteilung ist die gemäß Sensibilisierungsweg. Die Diagnostik der Nahrungsmittelallergie umfasst eine sorgfältige Anamnese, Hauttestungen und den Nachweis allergenspezifischer IgE-Antikörper sowie standardisierte orale Provokationstestungen. Die zur Verfügung stehenden Hauttest-Extrakte sind oft instabil und wichtige Allergene fehlen darin, z.T. aufgrund des Extraktionsprozesses, z.T. weil die Einzelallergene der jeweiligen Nahrungsmittelallergen-Quelle noch nicht vollständig bekannt sind. Daher werden Nahrungsmittel zumeist roh an der Haut getestet, was allerdings aufgrund der Tatsache, dass über den jeweiligen Allergengehalt nichts bekannt ist bzw. dieser je nach Reifungsgrad variiert, riskant ist. Kreuzreaktionen sind häufig, aber nicht sicher vorhersehbar. Darüber hinaus können Nahrungsmittelallergene neu auftreten (neu importierte tropische Früchte: Novel Food) oder „versteckt“ vorkommen durch fehlende Deklaration bzw. Deklarationspflicht und Kontamination während des Herstellungsprozesses. Die Identifizierung, Klonierung und Produktion rekombinanter allergener Proteine sowie die Synthese IgE-Epitop-darstellender Peptide hat eine komponentenaufgelöste Diagnostik möglich gemacht, indem IgE-Antikörper, die spezifisch für ein allergenes Protein oder sogar ein sequenzielles Epitop sind, detektiert und quantifiziert werden können. Für pflanzliche Nahrungsmittelallergen-Quellen stehen einige Risikoallergene für schwere systemische Reaktionen bereits für die Labordiagnostik zur Verfügung: das Soja-Allergen Gly m 4 (Glycine maxima, Sojabohne), welches dem Majorallergen der Birke (Bet v 1, Betula verrucosa) sehr ähnlich ist (Bet-v-1-homologes Protein), das Erdnuss-Speicherprotein Ara h 2 (Arachis hypogaea), das Lipidtransfer-Protein (LTP) der Erdnuss Ara h 9, das Haselnuss-LTP Cor a 8 (Corylus avellana), das Pfir- 
sich-LTP Pru p 3 (Prunus persica) und das Weizenprotein Omega-5-Gliadin Tri a 19 (Triticum aestivum). Ungeeignet ist der Nachweis von IgG4 auf Nahrungsmittelallergene.

Risikofaktoren einer Nahrungsmittelallergie sind Erkrankungen aus dem atopischen Formenkreis, wie atopisches Ekzem, allergische Rhinokonjunktivitis und allergisches Asthma. Diese Patienten sind besonders gefährdet, eine Anaphylaxie auf ein Nahrungsmittel zu entwickeln. Zu den Augmentationsfaktoren zählen Alkohol, körperliche Belastung und Medikamente, hierunter insbesondere die nicht steroidalen Antiphlogistika.

Für die klassischen Nahrungsmittelallergene, wie Hühner- und Milcheiweiß, die eher im Kindesalter Ursache einer Nahrungsmittelallergie sein können, kann sich eine natürliche Toleranz entwickeln. Nach 1-2 Jahren Allergenkarenz im frühen Kindesalter sollte daher die entsprechende Diätempfehlung - je nach Schwere der Symptome ggf. stationär - per expositionem reevaluiert werden. Unter den Provokationstests gilt die doppelblinde, placebokontrolliert durchgeführte orale Nahrungsmittelprovokation als Goldstandard der Nahrungsmittelallergie-Diagnostik. Orale Provokationstests sollten generell in der symptomarmen und möglichst stabilen Phase der Erkrankung bzw. bei Erscheinungsfreiheit vorgenommen werden. Die Schwere der vorangegangenen klinischen Reaktion ist kein verlässlicher Hinweis für die Schwere einer Folgereaktion. Bedrohliche Soforttypreaktionen in der Vorgeschichte stellen eine Kontraindikation für orale Provokationstestungen dar. Eine allergenspezifische Immuntherapie mit Pollenextrakten sollte bei pollenassoziierter Nahrungsmittelallergie nur dann erfolgen, wenn eindeutige pollenbedingte Atemwegssymptome bestehen.

Interessenkonflikt: Die Autorin erklärt, dass kein Interessenkonflikt im Sinne der Richtlinien des International Committee of Medical Journal Editors (ICMJE; www.icmje.org ) besteht.

\section{Literatur}

1 Werfel T. Facharztwissen Nahrungsmittelallergie. J Dtsch Dermatol Ges 2008; 6: 573-585

2 Werfel T, Erdmann S, Fuchs T et al. Leitlinie: Vorgehen bei vermuteter Nahrungsmittelallergie bei atopischer Dermatitis. J Dtsch Dermatol Ges 2009; 7: 265-271

3 Gimenez-Arnau A, Maurer M, de la Cuadra J et al. Immediate contact skin reactions, an update of contact urticaria, contact urticaria syndrome and protein contact dermatitis - A never ending story. Eur J Dermatol 2010; 20: $552-562$

4 Bock SA, Munoz-Furlong A, Sampson HA. Further fatalities caused by anaphylactic reactions to food, 2001-2006. J Allergy Clin Immunol 2007; 119: 1016-1018

5 Henzgen M, Vieths S, Reese I et al. Nahrungsmittelallergien durch immunologische Kreuzreaktionen. Leitlinie der DGAl und des ÄDA. Allergo J 2005; 14: 48 - 59

6 Hompes S, Scherer $K$, Köhli A et al. Nahrungsmittelanaphylaxie: Daten aus dem Anaphylaxie-Register. Allergo J 2010; 19: 234-243

7 Pichler W. IgE-vermittelte Nahrungsmittelallergien. Klassifikation basierend auf dem Sensibilisierungsweg. Allergologie 1998; 21: $441-450$

8 Wüthrich B. Contact urticaria and protein contact dermatitis to food. In: Marone G, Hrsg. Clinical Immunology and Allergy in Medicine. Neapel: JGC Editions; 2003: 175 $-182$

9 Fiedler EM, Zuberbier T, Worm M. A combination of wheat flour, ethanol and food additives inducing FDEIA. Allergy 2002; 57: 1090 - 1091

10 Raulf-Heimsoth M, Stark R, Sander I et al. Anaphylactic reaction to apple juice containing acerola: cross-reactivity to latex due to prohevein. J Allergy Clin Immunol 2002; 109: $715-716$

11 Breiteneder $H$. Die Klassifizierung der pflanzlichen Nahrungsmittelallergene. Allergologie 2009; 32: 375-382

12 King TP, Hoffmann D, Lowenstein H et al. Allergen nomenclature. WHO/IUIS Allergen Nomenclature Subcommittee. Bull World Health Organ 1994; 72: 796-806

13 Bartra J. From pollinosis to digestive allergy. J Invest Allergol Clin Immunol 2009; 19 (1): 3-10

14 Kleine-Tebbe J, Vogel L, Crowell DN et al. Severe oral allergy syndrome and anaphylactic reactions caused by a Bet $v$ 1-related PR-10 protein in soybean SAM 22. J Allergy Clin Immunol 2002; 110: 797-804

15 Palacin A, Quirce S, Sánchez-Monge R et al. Allergy to kiwi in patients with baker's asthma: identification of potential cross-reactive allergens. Ann Allergy Asthma Immunol 2008; 101: 200-205

16 Commission Directive 2006/142/EC of 22 December 2006 amending Directive 2000/13/EC listing the ingredients which must under all circumstances appear on the labelling of foodstuffs. Official Journal of the European Union 2006; L368/110

17 Novel Food Verordnung: Verordnung (EG) Nr. 258/97 des Europäischen Parlaments und des Rates vom 27. Januar 1997 über neuartige Lebensmittel und neuartige Lebensmittelzutaten. http://eur-lex.europa.eu/LexUriServ/LexUriServ.do? uri=CELEX:31997R0258:DE:HTML Stand: 27.04.2011

18 Fine AJ. Hypersensitivity reaction to kiwi fruit (Chinese gooseberry, Actinidia chinensis). J Allergy Clin Immunol 1981; 68: 235-237

19 Jappe U, Vieths S. Lupine: A source of new as well as hidden food allergens? Molecular Nutrition and Food Research 2010; 54: 113-126

20 Brennecke S, Becker WM, Lepp U et al. Anaphylactic reaction to lupine flour. J Dtsch Dermatol Ges 2007; 5: 774 776

21 Kleinheinz A, Jappe U, Petersen A. Anaphylaktische Reaktionen auf Granatapfel: Identifizierung und Charakterisierung von IgE-reaktiven Komponenten. Allergologie 2010; 19: S18

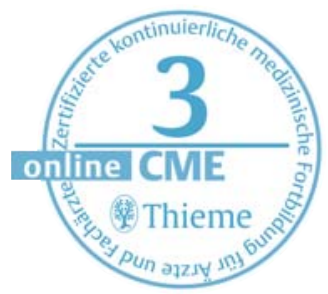


22 Niggemann B, Erdmann S, Fuchs T et al. Standardisierung von oralen Provokationstests bei Nahrungsmittelallergien. Allergo | 2006; 15: 262 - 270

23 Kleine-Tebbe J, Ballmer-Weber B, Beyer K et al. In-vitro-Diagnostik und molekulare Grundlagen von IgE-vermittelten Nahrungsmittelallergien. Leitlinie der Deutschen Gesellschaft für Allergie und Klinische Immunologie (DGAKI), des Ärzteverbandes Deutscher Allergologen (ÄDA) und der Gesellschaft für Pädiatrische Allergologie und Umweltmedizin (GPA). Allergologie 2009; 32: 177 194

24 Kleine-Tebbe J, Meißner AM, Jappe U et al. Allergenfamilien und molekulare Diagnostik von IgE-vermittelten Nahrungsmittelallergien: Von der Theorie zur Praxis. Allergo J 2010; 19: 251 - 264

25 Stapel SO, Asero R, Ballmer-Weber BK et al. Testing for IgG4 against foods is not recommended as a diagnostic tool: EAACI Task Force Report. Position Paper. Allergy 2008; 63: $793-796$

26 Renz H, Biedermann T, Bufe A et al. In vitro-Allergiediagnostik. Leitlinie der Deutschen Gesellschaft für Allergologie und Klinische Immunologie (DGAKI) unter Beteiligung des Ärzteverbandes Deutscher Allergologen (ÄDA), der Gesellschaft für Pädiatrische Allergologie und Umweltmedizin (GPA) und der Deutschen Dermatologischen Gesellschaft (DDG). Allergo J 2010; 19: 110 128

27 Jappe U. Diagnostic reagents for type-l-allergy - What criteria should be applied to validation? Arb Paul Ehrlich Inst Bundesamt Sera Impfstoffe; Frankf a. M.: 2009; 96: $135-145$

28 Henzgen M, Ballmer-Weber BK, Erdmann S et al. Hauttestungen mit Nahrungsmittelallergenen. Leitlinie der Arbeitsgruppe Nahrungsmittelallergie der Deutschen Gesellschaft für Allergologie und Klinische Immunologie (DGAKI), dem Ärzteverband Deutscher Allergologen (ÄDA), und der Gesellschaft für Pädiatrische Allergologie (GPA), zusammen mit der Schweizerischen Gesellschaft für Allergologie und Immunologie. Allergologie 2008; 31: $274-280$

29 Rueff F, Bergmann KC, Brockow K et al. Hauttests zur Diagnostik von allergischen Soforttypreaktionen. Allergo J 2010; 19: $402-415$

30 Werfel $T$, Reese I. Diätetik in der Allergologie. Diätvorschläge, Positionspapiere und Leitlinien zu Nahrungsmittelallergie und anderen Unverträglichkeiten. 3. Aufl. München, Orlando: Dustri; 2010

31 Erdmann SM, Werfel T. Hematogenous contact eczema induced by foods. Hautarzt 2006; 57: 116-120
32 Jankiewicz A, Aulepp H, Baltes W et al. Allergic sensitization to native and heated celery root in pollen-sensitive patients investigated by skin test and lgE binding. Int Arch Allergy Immunol 1996; 11: 268-278

33 Dannaeus A, Inganas M. A follow-up study of children with food allergy. Clinical course in relation to serum lgE- and lgG-antibody levels to milk, egg and fish. Clin Allergy 1981; 11: 533-539

34 Bock SA. The natural history of food sensitivity. J Allergy Clin Immunol 1982; 69: 173-177

35 Host A, Halken S. A prospective study of cow milk allergy in Danish infants during the first 3 years of life. Clinical course in relation to clinical and immunological type of hypersensitivity reaction. Allergy 1990; 45: 587-596

36 Ring J, Brockow K, Duda D et al. Akuttherapie anaphylaktischer Reaktionen (AWMF-Leitlinien-Register 061/025). Allergo J 2007; 16: 420-434

37 Lepp U, Ballmer-Weber B, Beyer $K$ et al. Therapiemöglichkeiten bei der IgE-vermittelten Nahrungsmittelallergie. Allergologie 2010; 33: 347-356

38 Tryba M, Ahnefeld FW, Barth j et al. Akuttherapie anaphylaktoider Reaktionen. Allergo J 1994; 3: 211 - 224

39 Tryba M. Therapie des anaphylaktischen Schocks. Notfallmedizin 1997; 23: $238-244$

40 Nelson HS, Lahr J, Rule R et al. Treatment of anaphylactic sensitivity to peanuts by immunotherapy with injections of aqueous peanut extract. J Allergy Clin Immunol 1997; 99: $744-751$

41 Oppenheimer J], Nelson HS, Bock A et al. Treatment of peanut allergy with rush immunotherapy. J Allergy Clin Immunol 1992; 90: 256-262

42 Enrique E, Pineda F, Malek T et al. Sublingual immunotherapy for hazelnut food allergy: a randomized, doubleblind, placebo-controlled study with a standardized hazelnut extract. I Allergy Clin Immunol 2005; 116: 1073 - 1079

43 Meglio P, Giampietro PG, Gianni S et al. Oral desensitization in children with immunoglobulin E-mediated cow's milk allergy-follow-up at $4 \mathrm{yr}$ and 8 months. Pediatr Allergy Immunol 2008; 19: 412-419

44 Petriarca G, Nucera E, Roncallo C et al. Oral desensitizing treatment in food allergy: clinical and immunological results. Aliment Pharmacol Ther 2003; 17: 459-465

45 Leung DYM, Sampson HA, Yinginger JW et al. Effect of antiIgE therapy in patients with peanut allergy. $\mathrm{N}$ Engl J Med 2003; 348 (11): 986-993

46 Jappe U, Raulf-Heimsoth $M$. Kreuzreagierende Kohlenhydratdeterminanten („cross-reactive carbohydrate determinants" $=(C D)$ und ihre Bedeutung für die Allergiediagnostik. Allergologie 2008; 31: 82-90

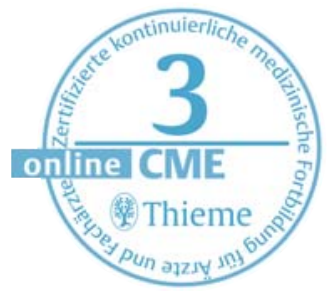




\section{CME-Fragen Nahrungsmittelallergie: Klinik, Diagnostik und Therapie}

1 Welche Antwort ist falsch?

Typische Allergene des Kleinkindesalters sind Hühnereiweiß

Milcheiweiß

Kern- und Steinobst

Erdnuss

Soja

2 Welche Antwort ist falsch?

Zu den Augmentationsfaktoren einer Nahrungsmittelallergie zählen

A Alkohol

B körperliche Belastung

C nicht steroidale Antiphlogistika

D Pollensaison

E Antibiotika

3 Welche Antwort trifft nicht zu?

Birkenpollenallergiker reagieren im Sinne der Kreuzreaktion häufig auf den Genuss von

A Baumnüssen

B Kern-/Steinobst

C Soja

D Banane

E Karotte

4 Welche Antwort ist richtig?

Das Majorallergen der Birke

gehört zu der Familie der Profiline.

B gehört zu den kreuzreaktiven Kohlenhydratdeterminanten.

C ist verantwortlich für Kreuzreaktionen zwischen Baumpollen und Nahrungsmitteln.

D ist ein verstecktes Allergen.

E ist der bedeutsamste Bestandteil von Novel Food.

5 Welche Aussage zu In-vitro-Untersuchungen bei Nahrungsmittelallergie trifft zu?

A Der Nachweis allergenspezifischer IgE-Antikörper beweist eine Nahrungsmittelallergie und ersetzt zeitaufwendige orale Provokationstestungen.

B Zelluläre Verfahren zum indirekten Nachweis einer IgEmediierten Reaktion sind einfach und können routinemäßig eingesetzt werden.

C Ein geeignetes Verfahren im Rahmen der Nahrungsmittelallergie-Diagnostik ist die Elektroakupunktur.

D Die Bestimmung von spezifischem IgG4 ist der IgEBestimmung gleichwertig und kann ersatzweise verwendet werden, sofern ein Labor nicht über IgE-Nachweismethoden verfügt.

E Die Konzentrationen allergenspezifischer IgE-Antikörper zeigen eine Sensibilisierung an, sind allerdings nicht beweisend für das Vorliegen einer Allergie und sollten daher nicht als alleinige Grundlage für Diätentscheidungen verwendet werden.
6 Welche Aussage trifft zu?

A Die häufigste Todesursache bei Nahrungsmittelallergie ist der anaphylaktische Schock.

B Die Erdnussallergie ist eine baumpollenassoziierte Nahrungsmittelallergie, löst daher zumeist eher harmlose oropharyngeale Symptome aus und kann in geröstetem Zustand gefahrlos verzehrt werden.

C Patienten mit atopischen Erkrankungen haben in der Regel kein hohes Risiko für Nahrungsmittelallergien.

D Latexallergiker können auch auf Obst und Gemüse reagieren.

E Aus dem Schweregrad der allergischen Reaktion auf ein Nahrungsmittel kann zuverlässig auf die Schwere von Folgereaktionen auf dieses Nahrungsmittel geschlossen werden.

7 Welche Aussage für Hauttestungen zur Diagnose einer Nahrungsmittelallergie trifft zu?

A Da kommerziell erhältliche NahrungsmittelallergenExtrakte oft labil sind, ist die Positivtestung auf ein natives Nahrungsmittel beweisend für das Vorliegen einer Allergie darauf.

B Die Testung mit Rohmaterial (Prick-zu-Prick-Testung) ist vollkommen ungefährlich.

C Reibtest und Scratch-Test sind ein wertvoller Ersatz für den Prick-Test.

D Der Atopie-Patch-Test mit Kuhmilch kann zur Diagnostik eines durch den Genuss von Milch verschlechterten atopischen Ekzems eingesetzt werden.

E Für den Erhalt der Allergenität von Nahrungsmitteln ist essenziell, bei Rohmaterialien nur tiefgefrorene Ware zu verwenden.

8 Welche Aussage trifft zu?

A Die pollenassoziierten Nahrungsmittelallergien treten vornehmlich im Kindesalter auf.

B Ein Allergen pflanzlichen Ursprungs, welches nicht mit Nahrungsmittelallergien assoziiert ist, ist Latex.

C Die häufigsten Nahrungsmittelallergien in Mitteleuropa sind die vom Typ C.

D Bedrohliche Soforttyp-Reaktionen nach Genuss von Nahrungsmitteln in der Vorgeschichte stellen die wichtigste Indikation für orale Provokationstestungen dar.

E Allergien gegen Hühner- oder Milcheiweiß im Kindesalter können sich später zurückbilden. 
9 Welche Aussage zur Therapie der Nahrungsmittelallergie trifft zu?

A Allergenkarenz ist die einzige hinsichtlich ihrer Effektivität bewiesene Therapieform bei Nahrungsmittelallergie.

B Der Nachweis von IgG-Antikörpern gegen Nahrungsmittel ist eine wertvolle Methode, individuelle Diäten zusammenzustellen.

C Die spezifische orale Toleranzinduktion mit Nahrungsmitteln ist aufgrund ihres Langzeiteffekts die Therapieoption der Wahl.

D Eine allergenspezifische Immuntherapie der Nahrungsmittelallergie ist ein etabliertes Verfahren.

E Nach der Diagnose einer Nahrungsmittelallergie sollte sofort medikamentös interveniert werden.
10 Welche Aussage trifft nicht zu?

Zur In-vitro-Nahrungsmittelallergie-Diagnostik ungeeignete Verfahren sind:

A Bioresonanz und Kinesiologie

B allergenspezifischer IgE-Antikörpernachweis

C Elektroakupunktur

D Lymphozyten-Transformationstest

E nahrungsmittelspezifisches IgG und IgG4

\section{CME-Fortbildung mit der Aktuellen Dermatologie}

Zertifizierte Fortbildung Hinter der Abkürzung CME verbirgt sich „continuing medical education“, also kontinuierliche medizinische Fort- und Weiterbildung. Zur Dokumentation der kontinuierlichen Fortbildung der Ärzte wurde das Fortbildungszertifikat der Ärztekammern etabliert. Hauptzielgruppe für das Fortbildungszertifikat sind Ärzte mit abgeschlossener Facharztausbildung, die im 5-jährigen Turnus einen Fortbildungsnachweis erbringen müssen. Es ist jedoch auch für Ärzte im Praktikum bzw. in der Facharztweiterbildung gedacht.

Die Fortbildungseinheit In den einheitlichen Bewertungskriterien der Bundesärztekammer ist festgelegt: „Die Grundeinheit der Fortbildungsaktivitäten ist der Fortbildungspunkt. Dieser entspricht in der Regel einer abgeschlossenen Fortbildungsstunde (45 Minuten)“. Für die erworbenen Fortbildungspunkte muss ein Nachweis erbracht werden. Hat man die erforderliche Anzahl von 250 Punkten gesammelt, kann man das Fortbildungszertifikat bei seiner Ärztekammer beantragen, welches man wiederum bei der KV (niedergelassene Ärzte) oder bei seinem Klinikträger (Klinikärzte) vorlegen muss.

Anerkennung der CME-Beiträge Die Fortbildung in der Aktuellen Dermatologie wurde von der Nordrheinischen Akademie für ärztliche Fort- und Weiterbildung für das Fortbildungszertifikat anerkannt, das heißt, die Vergabe der Punkte kann direkt durch die Thieme Verlagsgruppe erfolgen. Die Fortbildung in der Aktuellen Dermatologie gehört zur Kategorie „strukturierte interaktive Fortbildung“. Entsprechend einer Absprache der Ärztekammern werden die von der Nordrheinischen Akademie für ärztliche Fort- und Weiterbildung anerkannten Fortbildungsveranstaltungen auch von den anderen zertifizierenden Ärztekammern anerkannt.
Datenschutz Ihre Daten werden ausschließlich für die Bearbeitung dieser Fortbildungseinheit verwendet. Es erfolgt keine Speicherung der Ergebnisse über die für die Bearbeitung der Fortbildungseinheit notwendige Zeit hinaus. Die Daten werden nach Versand der Testate anonymisiert. Namens- und Adressangaben dienen nur dem Versand der Testate. Die Angaben zur Person dienen nur statistischen Zwecken und werden von den Adressangaben getrennt und anonymisiert verarbeitet.

Teilnahme Jede Ärztin und jeder Arzt soll das Fortbildungszertifikat erlangen können. Deshalb ist die Teilnahme am CME-Programm der Aktuellen Dermatologie nicht an ein Abonnement geknüpft! Die Teilnahme ist im Internet unter http://cme.thieme. de möglich. Man muss sich registrieren, wobei die Teilnahme an Fortbildungen abonnierter Zeitschriften ohne Zusatzkosten möglich ist.

Teilnahmebedingungen Für eine Fortbildungseinheit erhalten Sie 3 Fortbildungspunkte im Rahmen des Fortbildungszertifikates. Hierfür müssen 70\% der Fragen richtig beantwortet sein.

CME-Fortbildung für Nicht-Abonnenten Teilnehmer, die nicht Abonnenten der Aktuellen Dermatologie sind, können für die Internet-Teilnahme dort direkt ein Guthaben einrichten, von dem pro Teilnahme ein Unkostenbeitrag abgebucht wird.

Teilnahme online unter http://cme.thieme.de 